\title{
EDUCAÇÃO EM SAÚDE PARA A \\ CRIANÇA/JOVEM/FAMÍLIA: NECESSIDADES \\ FORMATIVAS DOS ENFERMEIROS
}

\author{
HEALTH EDUCATION FOR THE \\ CHILD/ADOLESCENT/FAMILY: \\ NURSES' TRAINING NEEDS
}

\section{EDUCACIÓN EN SALUD PARA LOS NIÑOS/JÓVENES/FAMILIA: NECESIDADES FORMATIVAS DE LAS ENFERMERAS}

\author{
Anabela Fonseca Pereira ${ }^{1}$ \\ Joaquim José Jacinto Escola ${ }^{2}$ \\ Carlos Manuel Torres Almeida ${ }^{3}$
}

\begin{abstract}
Como citar este artigo: Pereira AF, Escola JJJ, Almeida CMT. Educação em Saúde para a criança/jovem/família: necessidades formativas dos enfermeiros. Rev baiana enferm. 2020;34:e35273.

Objetivo: identificar necessidades de formação dos enfermeiros em Educação em Saúde para a criança/jovem/família. Método: pesquisa quantitativa aplicada a uma amostra de 311 enfermeiros que realizavam Educação em Saúde para a criança/jovem/família em dois centros hospitalares e dois agrupamentos de atenção primária da província de Trás-os-Montes e Alto Douro no segundo semestre de 2018. Resultados: entre os inquiridos, 84,9\% (n=264) não seguia nenhum modelo/teoria de Educação em Saúde; 66,2\% (n=206) não frequentou nenhuma formação no âmbito da Educação em Saúde nos últimos cinco anos; 98,7\% (n=307) considerou que a formação em Educação em Saúde é importante para o seu desenvolvimento profissional; e 93,6\% (n=291) referiu necessidade de formação específica no âmbito da Educação em Saúde, nomeadamente em "técnicas de comunicação" (50,5\%; n=157) e "estratégias técnico-pedagógicas" (50,5\%; n=157). Conclusão: os enfermeiros sentiam necessidade de formação em modelos/ teorias, técnicas de comunicação e estratégias técnico-pedagógicas de intervenção na Educação em Saúde.
\end{abstract}

Descritores: Promoção da Saúde. Educação em Saúde. Enfermagem. Saúde da Criança. Saúde do Adolescente.

Objective: to identify nurses' training needs in Health Education for the child/adolescent/family. Method: quantitative survey applied to a sample of 311 nurses who performed Health Education for the child/adolescent/family in two hospital centers and two primary care groupings in the province of Trás-os-Montes e Alto Douro in the second half of 2018. Results: among the respondents, 84.9\% ( $n=264)$ did not follow any model/theory of bealth education; $66.2 \%(n=206)$ did not attend any training in the context of Health Education in the past five years; $98.7 \%(n=307)$ considered that the training in Health Education is important for their professional development; and 93.6\% ( $n=291$ ) reported the need for specific training in the field of Health Education, particularly in "communication techniques" (50.5\%; $n=157)$ and "technical-pedagogical strategies" (50.5\%; n=157). Conclusion: the nurses felt the need for

Enfermeira. Especialista em Saúde Infantil e Pediatria. Doutora em Ciências da Educação. Enfermeira na Unidade Local de Saúde do Nordeste, EPE. Vila Real, Portugal. anabelapereira83@gmail.com. http://orcid.org/0000-0002-8588-08।4.

Graduado em Filosofia. Doutor em Ciências Educacionais. Professor Auxiliar da Universidade de Trás-Os-Montes e Alto Douro. Vila Real, Portugal. http://orcid. org/0000-0002-6676-6928.

Graduado em Enfermagem. Doutor em Bioética. Professor Adjunto da Universidade de Trás-Os-Montes e Alto Douro. Vila Real, Portugal. http://orcid.org/00000002-4497-4267. 
training in models/theories, communication techniques and technical-pedagogical strategies of intervention in Health Education.

Descriptors: Health Promotion. Health Education. Nursing. Child Health. Adolescent Health.

Objetivo: identificar las necesidades formativas de las enfermeras en la Educación en Salud de los niños/jóvenes/ familia. Método: estudio cuantitativo aplicado a una muestra de 311 enfermeras que realizan en la Educación en Salud de los niños/jóvenes/familia en dos centros hospitalarios y dos grupos de atención primaria en la provincia de Trás-os-Montes e Alto Douro, en el segundo semestre de 2018. Resultados: entre los encuestados, el 84,9\% ( $n=264)$ no sigue ninguna teoría/modelo de Educación en Salud; el 66,2\% (n=206) no realizó ninguna formación en el contexto de la Educación en Salud en los últimos cinco años; el 98, 7\% (n=307) considera que la formación en Educación en Salud es importante para su desarrollo profesional; y el 93,6\% ( $n=291)$, destacó la necesidad de formación específica en el campo de la Educación en Salud, especialmente en "técnicas de comunicación" (50,5\%; $n=157)$ y "estrategias técnico-pedagógicas" (50,5\%; n=157). Conclusión: las enfermeras consideraron la necesidad de formación en teorías y modelos, técnicas de comunicación y estrategias técnico-pedagógica de intervención en Educación en Salud.

Descriptores: Promoción de la Salud. Educación en Salud. Enfermería. Salud del Niño. Salud del Adolescente.

\section{Introdução}

A promoção da saúde tem sido assumida como um amplo paradigma, em que o indivíduo é participante ativo nas estratégias educativas que promovem a autonomia e a tomada de decisão com vista à mudança de comportamentos. Por isso, a Educação em Saúde (ES) é uma prática transversal e intersectorial na sociedade ${ }^{(1)}$. Nesse sentido, também o investimento na saúde ao longo do ciclo de vida, por meio da capacitação dos cidadãos, é parte integrante do quadro de referência para as políticas europeias de saúde ${ }^{(2)}$.

Entretanto, devido às rápidas transformações sociais, os cuidados de Enfermagem são cada vez mais culturalmente diversificados, fazendo com que o enfermeiro deva ter determinadas competências e habilidades adquiridas na formação continuada ${ }^{(3)}$. Embora o papel de educador nem sempre seja valorizado por parte do enfermeiro, este assume um desempenho importante em $\mathrm{ES}^{(4)}$, já que não se limita a preencher lacunas no serviço prestado à comunidade. Este profissional destaca-se como planejador de ações transformadoras que provocam mudanças no seu contexto profissional e social ${ }^{(5)}$.

No contexto da prática de Enfermagem em ES voltada à criança/jovem/família, busca-se promover a saúde familiar e aumentar o bem-estar da unidade familiar (pais e criança/jovem). Esta prática revela a importância do enfermeiro como agente educador e com funções de orientação à criança/jovem/família, de forma a responder às suas necessidades ${ }^{(6)}$.

Tendo em conta que a promoção da saúde é uma preocupação atual e um tema central constituinte da agenda política de muitos governos ${ }^{(7)}$, é da responsabilidade dos enfermeiros conceber e planejar programas de intervenção no âmbito da ES. Esta prática está descrita nos padrões de qualidade do exercício profissional de Enfermagem ${ }^{(8)}$ e prevê que os enfermeiros são essenciais em saúde, sendo fundamental assegurar que a educação em Enfermagem capacite-os para trabalhar em parceria com outros profissionais e responda às necessidades dos sistemas de saúde ${ }^{(9)}$.

Este estudo de investigação teve como objetivo identificar necessidades formativas dos enfermeiros em ES para a criança/jovem/família.

\section{Método}

Pesquisa quantitativa e transversal realizada em dois centros hospitalares e dois agrupamentos de centros de saúde da província de Trás-os-Montes e Alto Douro, num total de 4 serviços de internamento hospitalar pediátrico e 29 centros de saúde - Centro Hospitalar de 
Trás-os-Montes e Alto Douro (EPE), Agrupamento de Centros de Saúde de Trás-os-Montes Alto Tâmega e Barroso, Agrupamento de Centros de Saúde do Douro I - Marão e Douro Norte, Unidade Local de Saúde do Nordeste (EPE) -, durante o segundo semestre de 2018. Optou-se pela escolha de uma amostragem não probabilística de conveniência.

Os critérios de inclusão da população alvo foram: ser profissional de Enfermagem a exercer a sua atividade há mais de 6 meses nos serviços de internamento hospitalar pediátrico; ser profissional de Enfermagem a exercer a sua atividade há mais de 6 meses nos cuidados de saúde primários cuja atividade passe por realizar consultas de saúde infantil e juvenil; e aceitação dos enfermeiros em participar do estudo. A amostra foi constituída por 311 enfermeiros $(78,5 \%$ da população em estudo).

Para a coleta de dados foi construído e utilizado um inquérito por questionário, que foi aplicado após autorização dos responsáveis máximos das instituições participantes do estudo e parecer favorável das respetivas comissões de ética (Administração Regional de Saúde do Norte: Parecer no 124/2018; Centro Hospitalar de Trás-os-Montes e Alto Douro, EPE: Parecer nº256/2018; Unidade Local de Saúde do Nordeste, EPE: Parecer $\mathrm{n}^{\mathrm{o}}$ 00316/2018). Foi igualmente assegurado que os questionários distribuídos integrassem o consentimento informado, sendo explicado aos participantes deste estudo que a sua participação não traria riscos e que tinham a plena liberdade de aceitação ou abandono do estudo, sem restrições ou consequências. Foi-lhes dada garantia de que haveria confidencialidade no que respeita à preservação dos dados recolhidos, que seriam exclusivamente utilizados para a pesquisa. A privacidade foi igualmente respeitada e assegurado o anonimato dos participantes.

Os dados recolhidos foram analisados no programa estatístico Statistical Package for the Social Sciences (SPSS 22.0). Para analisar as caraterísticas sociodemográficas, recorreu-se à análise descritiva (distribuições de frequências, medidas de tendência central e medidas de dispersão).
Para as associações entre as variáveis, fez-se uso da inferência estatística ${ }^{(10)}$. O teste Kolmogorov-Smirnov revelou que a amostra não apresentava uma distribuição normal e recorreu-se ao teste não paramétrico Qui-Quadrado $(\chi)^{(10)}$ por simulação de Monte Carlo. Para todos os testes estatísticos, adotou-se um nível de significância de $0,05(\mathrm{p}<0,05)$.

\section{Resultados}

Em relação às caraterísticas sociodemográficas e profissionais, os resultados mostraram que, para o número total de casos válidos $(n=311)$, 89,1\% ( $n=277)$ dos enfermeiros eram do gênero feminino e 10,9\% $(n=34)$ do gênero masculino; a moda da idade situou-se no intervalo dos 40 aos 50 anos de idade e a do tempo de exercício profissional situou-se no intervalo dos 15 aos 20 anos; 56,6\% $(n=156)$ dos inquiridos possuíam somente a Licenciatura e 39\% $(n=121)$ tinham o curso de Pós-Licenciatura de Especialização; 13,5\% ( $\mathrm{n}=42)$ exerciam a sua atividade profissional no hospital e $86,6 \%(n=269)$, nos cuidados de saúde primários.

Das variáveis que permitiram retratar as necessidades formativas dos enfermeiros no âmbito da ES para a criança/jovem/família, os resultados mostraram que, em relação ao modelo/ teoria adotado para a realização dessa prática, $84,9 \%(n=264)$ dos inquiridos referiu não seguir nenhum modelo/teoria e somente 15,1\% ( $n=47)$ afirmou seguir algum modelo/teoria. Os modelos/teorias mais apontados pelos 15,1\% $(n=47)$ dos inquiridos foram: normas de Direção-Geral da Saúde e o "Plano Nacional de Saúde Infantil e Juvenil".

Relativamente à frequência de formação no âmbito da ES, nos últimos cinco anos, verificou-se que 66,2\% (n=206) não cursou nenhuma formação e 33,8\% (n=105) afirmou ter frequentado. A maioria dos enfermeiros que participou de formação específica em ES tinha entre 40 e 50 anos de idade.

Quando questionados se consideravam importante a formação em ES para o desenvolvimento profissional como enfermeiros, 
$98,7 \%(n=307)$ considerou que sim e 1,3\% (n=4) considerou não ser importante.

Quando inquiridos sobre a necessidade de formação específica no âmbito da ES, constatou-se que 93,6\% (n=291) dos enfermeiros sentiam essa necessidade. Os tópicos das necessidades de formação específica em ES para a criança/jovem/família mais apontados foram as "técnicas de comunicação" $(50,5 \% ; n=157)$ e as "estratégias técnico-pedagógicas" (50,5\%; n=157) (Tabela 1).

Tabela 1 - Distribuição dos enfermeiros inquiridos segundo o âmbito das necessidades de formação em Educação em Saúde. Trás-os-Montes e Alto Douro, Região Norte de Portugal, Portugal - 2018. $(\mathrm{N}=311)$

\begin{tabular}{|c|c|c|}
\hline Formação específica no âmbito da Educação em Saúde & $\mathbf{n}$ & $\%$ \\
\hline Planificação de atividades de Educação em Saúde & 119 & 38,8 \\
\hline Desenvolvimento de atividades de Educação em Saúde & 145 & 46,6 \\
\hline Processos de ensino-aprendizagem & 132 & 42,4 \\
\hline Revisão da literatura & 100 & 32,2 \\
\hline Técnicas de comunicação & 157 & 50,5 \\
\hline Estratégias técnico-pedagógicas & 157 & 50,5 \\
\hline Aquisição de competências formativas & 135 & 43,4 \\
\hline Aquisição de competências relacionais & 77 & 24,8 \\
\hline Avaliação de necessidades da criança/jovem/família & 125 & 40,2 \\
\hline Compreensão de teorias e modelos de Educação em Saúde & 151 & 48,6 \\
\hline Conhecimento de práticas de desenvolvimento de Educação em Saúde & 79 & 25,4 \\
\hline Conhecimento sobre obter, tratar e analisar dados & 75 & 24,1 \\
\hline Formação em tecnologias de informação & 65 & 20,9 \\
\hline Construção de indicadores & 94 & 30,2 \\
\hline
\end{tabular}

Fonte: Elaboração própria.

A análise da associação entre o reconhecimento da formação, no âmbito da ES, como importante para o desenvolvimento profissional, não revelou a existência de diferenças estatisticamente significativas com as "habilitações académicas/profissionais" ( $\left.\chi^{2}=1,534 ; \mathrm{df}=4 ; p=0,683\right)$; "tempo de exercício profissional" $\left(\chi^{2}=8,972\right.$; $\mathrm{df}=5 ; p=0,128)$, e "local de exercício profissional" $\left(\chi^{2}=0,633 ; \quad \mathrm{df}=1 ; \quad p=0,648\right)$. Entretanto, verificou-se uma relação com a "idade" $\left(\chi^{2}=22,202\right.$; $\mathrm{df}=4 ; p=0,007)$, sendo esse efeito no intervalo dos 50 a 60 anos.

A análise da associação entre a necessidade de formação específica no âmbito da ES e as variáveis "idade" $\left(\chi^{2}=4,315 ; \mathrm{df}=4 ; p=0,301\right)$, "habilitações académicas/profissionais" $\left(\chi^{2}=3,082\right.$; $\mathrm{df}=4 ; p=0,475)$, e "tempo de exercício profissional" $\left(\chi^{2}=5,4444 ; \mathrm{df}=5 ; p=0,318\right)$ não revelaram a existência de diferenças estatisticamente significativas. Contudo, verificou-se que a variável "local de exercício profissional" influencia a necessidade de formação específica no âmbito da ES $\left(\chi^{2}=12,845 ; \mathrm{df}=1 ; p=0,002\right)$, encontrando-se o efeito no âmbito dos cuidados de saúde primários. Isto é, entre os enfermeiros dos cuidados de saúde primários, existe maior necessidade de formação específica em ES, pois, entre esses, observa-se um número de respostas superior ao esperado $(3,6)$ e, entre os enfermeiros que exercem atividade profissional no internamento hospitalar pediátrico, um número de respostas inferior ao esperado $(-3,6)$ (Tabela 2). 
Tabela 2 - Resultados do teste Qui-Quadrado por simulação de Monte Carlo aplicado às variáveis "local de exercício profissional" e "necessidades formativas no âmbito da Educação em Saúde". Trás-os-Montes e Alto Douro, Região Norte de Portugal, Portugal, 2018. (N=311)

\begin{tabular}{l|l|c|c|c|c|c}
\hline \multirow{2}{*}{ Local onde exerce funções } & \multicolumn{3}{c|}{$\begin{array}{c}\text { Necessidade de formação no âmbito da } \\
\text { Educação em Saúde }\end{array}$} & \multicolumn{2}{c}{$\begin{array}{c}\text { Teste } \\
\text { Qui-Quadrado }\end{array}$} \\
\cline { 2 - 6 } & $\begin{array}{c}\text { Residuais } \\
\text { standartizados }\end{array}$ & Sim & Não & n & Valor & df \\
\hline Cuidados de Saúde Primários & Contagem & $257(1)$ & $12(2)$ & 269 & $12,845(1)$ & 1 \\
& Contagem esperada & 251,7 & 17,3 & 269,0 \\
& Resíduos ajustados & 3,6 & $-3,6$ & 42 \\
Unidade Hospitalar & Contagem & $34(1)$ & $8(2)$ & 42,0 \\
& Contagem esperada & 39,3 & 2,7 & 4,0 \\
& Resíduos ajustados & $-3,6$ & 3,6 & \\
& \multicolumn{5}{c}{ Razão de verossimilhança } & 9,455 \\
Associação Linear por Linear & 12,804 & 1 \\
\hline
\end{tabular}

Fonte: Elaboração própria.

Notas:

(1) 1 célula $(25,0 \%)$ esperavam uma contagem menor que 5. A contagem mínima esperada é 2,70.

(2) Computado apenas para uma tabela $2 \times 2$.

\section{Discussão}

Diante das atuais tendências, as necessidades de cuidados e a expectativa de que todas as crianças/jovens/famílias devem ter acesso a cuidados de Enfermagem que promovam a saúde em uma abordagem holística ${ }^{(8)}$, percebe-se que as intervenções de Enfermagem no âmbito da ES para a criança/jovem/família necessitam ser fundamentadas em teorias e/ou modelos. Todavia, a adoção de um modelo de ES pode não ser uma tarefa simples, não apenas porque, na literatura, foram encontrados diferentes teorias/modelos de ES, como também porque a formação em Enfermagem permanece predominantemente centrada no modelo biomédico ${ }^{(11)}$.

Tendo-se verificado que a maioria dos enfermeiros $(84,9 \% ; n=264)$ não seguia nenhum modelo/teoria durante a prática de ES para a criança/jovem/família, este resultado pode ser efetivamente fruto da diversidade de teorias/modelos de ES e/ou do desconhecimento dessas por parte dos enfermeiros. Reforçando essa ideia, verificou-se que, embora 98,7\% (n=307) dos enfermeiros considerasse a formação no âmbito da ES importante para o seu desenvolvimento profissional, apenas 33,8\% $(n=105)$ frequentou formação específica no âmbito da ES nos últimos cinco anos.

Por outro lado, podendo as abordagens de ES serem classificadas em três categorias - abordagem tradicional, abordagem de transição e abordagem moderna ${ }^{(12)}$-, se for considerado que os modelos/abordagens adotados pelos enfermeiros vão caracterizar a sua prática de ES, o fato de $84,9 \%(n=264)$ afirmar não seguir nenhuma teoria/modelo pode levar a pensar que os enfermeiros estão ligados a uma abordagem tradicional. Outro entendimento é apresentado em estudo que considera a possibilidade de os enfermeiros estarem ligados a quadros tradicionais de limitadas e limitadoras práticas de promoção da saúde, devido ao excesso de trabalho, inadequada educação e prática, além de foco no modelo biomédico ${ }^{(13)}$.

Uma vez que 93,6\% ( $n=291)$ dos inquiridos reconhece a necessidade de formação específica no âmbito da ES, e que o praticante de promoção da saúde adquire competências ao basear o seu conhecimento nos conceitos, princípios, valores éticos e teorias da Promoção da Saúde para as aplicar na prática ${ }^{(14)}$, vale pontuar a importância e necessidade de os enfermeiros terem acesso a formação que lhes permita a compreensão dos 
modelos/teorias subjacentes às abordagens em ES, garantindo o desenvolvimento de habilidades, atualização e fortalecimento de competências ${ }^{(15)}$.

Vários estudos sugerem a necessidade de reformulação dos processos formativos, pois o desenvolvimento de competências em promoção da saúde confere recursos cognitivos, habilidades e atitudes que dá resposta aos padrões profissionais da atuação em Promoção da Saúde ${ }^{(16)}$.

Nesse sentido, a preferência será o desenvolvimento desses processos formativos na instituição de saúde onde os profissionais exercem a sua atividade, para que haja lugar para uma reflexão individual e coletiva, uma partilha de experiências/saberes, e uma avaliação das práticas que potencie processos de melhoria contínua do exercício profissional da Enfermagem no âmbito da ES para a criança/jovem/família.

Verificou-se ainda que somente $33,8 \%(n=105)$ dos enfermeiros frequentaram formação específica no âmbito da ES nos últimos cinco anos. Este fato pode levar a pensar que os enfermeiros dão preferência à formação centrada na atualização de conhecimentos técnico-científicos. Melhor resultado obteve estudo ${ }^{(17: 89)}$ no qual $50 \%$ dos enfermeiros referiu ter feito "Formação de suporte à ES" nos últimos cinco anos.

Foi possível constatar também que 98,7\% $(n=307)$ dos enfermeiros considera que a formação no âmbito da ES é importante para o seu desenvolvimento profissional. Embora não se tenha encontrado diferenças estatisticamente significativas entre a importância da formação em ES para o desenvolvimento profissional e as habilitações académicas/profissionais, tempo de exercício e local profissional, verificou-se uma relação com a idade, sendo o efeito situado no intervalo de idades dos 50 aos 60 anos. Nesse sentido, pode-se deduzir que os enfermeiros mais novos valorizavam mais as técnicas e os procedimentos do que a prática de ES, o que poderá indicar a necessidade de maior aprofundamento teórico dessa prática na graduação da Enfermagem.

Pode-se também pensar na possibilidade de esse tema ser pouco valorizado pelas entidades responsáveis pela formação nas instituições de saúde. Entretanto, a formação dos profissionais que praticam ES, perante a atual realidade em constante mutação, tem de capacitar não só com os conhecimentos técnicos e científicos, sendo necessário um levantamento das necessidades por parte das instituições, para que evidenciem as suas potencialidades em ES. Assim, é igualmente importante motivar as instituições a promover formação que permita aprofundar e/ou aperfeiçoar conhecimentos, competências e atitudes/práticas profissionais no âmbito da ES para a criança/jovem/família, além de estratégias de intervenção em ES (planejamento, execução e avaliação). Até porque a prática de educar tem um carácter essencialmente reflexivo, em que se favorece a horizontalidade das relações entre profissionais e comunidade, o diálogo, a autonomia e a tomada de decisão ${ }^{(18)}$.

Paralelamente, 93,6\% (n=291) dos enfermeiros referiram sentir necessidade de frequentar formação específica no âmbito da prática de ES, nomeadamente em "técnicas de comunicação" (50,5\%; $\mathrm{n}=157)$ e "estratégias técnico-pedagógicas" (50,5\%; $\mathrm{n}=157)$. Isto pode ser indicativo do reconhecimento, por parte dos enfermeiros, da importância, complexidade e natureza dos processos de ES para a criança/jovem/família, da necessidade de rever/aprofundar conceitos inerentes a essa prática para implementar intervenções consistentes, e do aperfeiçoamento de competências para lidar com o binômio criança-jovem e família. Estudo sobre a identificação de fatores de implementação de práticas educativas em promoção da saúde verificou que os enfermeiros apontaram como fator influenciador dessa implementação a ausência de qualificação profissional em $\mathrm{ES}^{(19)}$.

Importa referir que a comunicação em saúde é igualmente entendida pelos enfermeiros como fundamental para a prática efetiva de ES, já que, em contexto pediátrico, constitui um elemento fulcral, influenciando a relação de parceria com a criança/jovem/família. Além disso, é uma das competências essenciais em promoção da saúde definidas pelo projeto pan-europeu Developing Competencies and Professional Standards for Health Promotion Capacity Building in Europe 
$(\text { CompHP })^{(14)}$ da Oficina Europeia da União Internacional de Promoção e Educação em Saúde. Estudo $^{(20)}$ salienta a necessidade de desenvolvimento da competência da comunicação durante a graduação em Enfermagem.

As competências em estratégias técnico-pedagógicas (estratégias de intervenção em ES) são igualmente importantes, já que a complexidade do comportamento humano implica ter a capacidade de planejamento, aplicação e avaliação de ferramentas técnico-pedagógicas, para que, de forma sistemática, promovam intervenções eficientes e eficazes com base na especificidade do binômio criança/jovem/família.

Em relação à necessidade de formação específica no âmbito da ES, e embora não tenha havido diferenças estatisticamente significativas relativas à idade, com o tempo de exercício profissional e com as habilitações académicas/profissionais, verificou-se a associação com o local de exercício profissional. Isto é, entre os enfermeiros de cuidados de saúde primários, verificou-se maior necessidade de formação específica em ES do que entre aqueles que exerciam a sua atividade profissional no internamento hospitalar pediátrico. Esta correlação com o local de trabalho pode ser o resultado do facto de a amostra dos enfermeiros a desempenhar a sua atividade profissional em cuidados de saúde primários ser mais expressiva $(86,5 \% ; n=269)$, e porque os estudos revelam que é nesse contexto que os enfermeiros desenvolvem mais frequentemente atividades de promoção da saúde ${ }^{(21)}$.

Ao comparar as práticas de promoção da saúde entre enfermeiros do contexto hospitalar e dos cuidados de saúde primários, estudo ${ }^{(22)}$ verificou que essas práticas diferiam com o contexto de trabalho. Isto é, nos cuidados de saúde primários, desenvolviam-se estratégias de promoção de estilos de vida saudáveis e referenciação de situação problemática de forma mais sistemática, enquanto que, no hospital, se identificava o problema e supervisionavam-se as atividades dos cuidados diretos e indiretos de forma mais meticulosa.

Já a pesquisa ${ }^{(23)}$ realizada com enfermeiros de contexto hospitalar, apontava para a necessidade de refletir sobre a formação do enfermeiro e as práticas de enfermagem no contexto hospitalar, de modo a conformá-las com os paradigmas atuais da promoção da saúde, que são assentes em uma visão holística do indivíduo, para que este seja inserido em um contexto dinâmico e participe ativamente da construção de seu projeto de vida saudável. O estudo aponta ainda que as falhas de formação vêm da formação dos cursos de base em enfermagem.

Evidências científicas relacionadas com os programas de intervenção no ensino superior mostram pouca relação com os requisitos dos contextos da promoção da saúde e poucos programas com os critérios de boas práticas de promoção da saúde ${ }^{(21)}$. Contudo, nos últimos anos, essas competências têm sido discutidas em contextos internacionais, e a literatura tem apontado para a existência de confusão entre conceitos nessas práticas, mesmo durante o processo formativo ${ }^{(24)}$.

Foram ainda realizadas várias recomendações sobre a importância de se proporcionar aos estudantes maior experiência clínica nos cuidados de saúde primários ${ }^{(25)}$. Fica, então, a ideia de maior aprofundamento teórico relativamente à promoção da saúde, quer na graduação de Enfermagem, quer na formação permanente, para que os enfermeiros não esqueçam a sua atualização técnico-científica e possam reconhecer o seu papel proativo quando da realização das intervenções em ES.

Os resultados obtidos no estudo realizado são úteis para o ensino da Enfermagem. Fica, portanto, a necessidade do aprofundamento teórico das teorias/modelos, conceitos, valores, princípios, estratégias comunicacionais e técnico-pedagógicas, para que se possa desenvolver um trabalho de potenciação das diversas competências, nomeadamente no âmbito da ES para a criança/jovem/família. Até porque, para a eficácia dos cuidados de Enfermagem, é importante que exista uma política de formação contínua promotora do desenvolvimento profissional.

Importa também referir a relevância da presente investigação para as instituições de saúde, uma vez que os resultados encontrados 
produziram evidências que podem servir de referência e se revelaram úteis para a melhoria e (re)formulação e/ou para o aperfeiçoamento das intervenções em ES para a criança/jovem/família. Logo, permite refletir crítica e estrategicamente essa área, assim como desenhar, implementar e avaliar o impacto das estratégias de ES nos diferentes contextos.

Considera-se ainda que o presente estudo permite alimentar a continuidade do debate em torno da ES realizada pelos enfermeiros para a criança/jovem/família, produzindo resultados promissores que reiterem a importância de se continuar a dar visibilidade à prática de ES realizada pelos enfermeiros na saúde da criança/jovem/família, permitindo manter processos de melhoria contínua da qualidade dos cuidados de Enfermagem.

Por último, como limitação do estudo, destaca-se o facto de restringir-se a uma área geográfica. Por isso, seria proveitoso, para a melhoria da prática de Enfermagem no âmbito da ES para a criança/jovem/família, que esta investigação fosse consolidada por meio de trabalhos mais abrangentes, de modo a obter resultados generalizados em âmbito nacional.

\section{Conclusão}

Os resultados da presente pesquisa revelaram que a maioria dos enfermeiros, nos últimos cinco anos, não frequentou formação específica em ES e não segue modelos/teorias associados à prática de ES. Contudo, eles consideram que a formação em ES é importante para o seu desenvolvimento profissional, assumindo necessidades formativas em ES para a criança/jovem/família, nomeadamente no âmbito das "técnicas de comunicação" e das "estratégias técnico-pedagógicas".

Recomenda-se o aprofundamento e aperfeiçoamento das competências e dos valores intrínsecos à prática de ES na graduação da Enfermagem e na formação permanente, para permitir a reflexão e a partilha de saberes sustentados em evidências, o desempenho do exercício profissional assente em processos de melhoria contínua dos padrões de qualidade, formar agentes promotores de saúde com ferramentas capazes de promover mudanças conscientes e sustentadas na criança/jovem/família, promover comportamentos potenciadores de saúde e contribuir para a visibilidade e melhoria da efetividade e da eficiência das intervenções de Enfermagem em ES para a criança/jovem/família.

\section{Colaborações:}

1 - concepção, projeto, análise e interpretação dos dados: Anabela Fonseca Pereira, Joaquim José Jacinto Escola e Carlos Manuel Torres Almeida;

2 - redação do artigo e revisão crítica relevante do conteúdo intelectual: Anabela Fonseca Pereira, Joaquim José Jacinto Escola e Carlos Manuel Torres Almeida;

3 - aprovação final da versão a ser publicada: Anabela Fonseca Pereira, Joaquim José Jacinto Escola e Carlos Manuel Torres Almeida.

\section{Referências}

1. Santos MP, Farre AGMC, Bispo MS, Sousa LB, Marinho DDT. Promoting adolescent sexual and reproductive health: peer education. Rev baiana enferm. 2017;31(3):e21505. DOI: http://dx.doi.org/ 10.18471/rbe.v31i3.21505

2. World Health Organization. Health Literacy. Solid Facts. [Internet] Copenhagen; 2013 [cited 2020 Jan 29]. Available from: http://www.euro.who. int/en/what-wedo/health-topics/environmentand-health/urbanhealth/publications/2013/ health-literacy.-thesolid-facts

3. Pacheco FC, Aguiar BRL, Araújo MC, Ramos CA, Protasio FC, Guilhem DB. Curricular analysis of bioethics teaching in undergraduate nursing courses in brazil. Rev baiana enferm. 2019;33:e27684. DOI: http://dx.doi.org/10.18471/rbe.r33.27684

4. Arnemann CT, Lavich CRP, Terra MG, Mello AL, Raddatz M. Health education and permanent education: actions integrating the educational process of nursing. Rev baiana enferm. 2019;32:e24719. DOI: http://dx.doi.org/10.158471/ rbe.v32.24719

5. Mayer BLD, Saioron I, Bruggmann MS. Professional health education in Brazil: a reflection in the context 
of nursing. Rev Bras Educ Saúde. 2019;9(4):1-9. DOI: http://dx.doi.org/10.18378/rebes.v9i3.6480

6. Silva FB, Gondim EC, Henrique NCP, Fonseca LMM, Mello DF. Educational intervention involving young mothers: gaining knowledge on childcare. Acta paul enferm. 2018 Mar;31(1):32-8. DOI: http://dx.doi.org/10.1590/ 1982-0194201800006

7. Bonito J, Oliveira H. A promoção e Educação para a Saúde com Crianças e Adolescentes nas escolas de Portugal. In: Franceschini SCC, Ribeiro SAV, Priore SE, Novaes JF, organizadores. Nutrição e Saúde da Criança. Rio de Janeiro: Rubio; 2019. p. 521-46.

8. Miranda FBG, Mazzo A, Pereira-Junior GA. Construction and validation of competency frameworks for the training of nurses in emergencies. Rev Latino-Am Enfermagem. 2018 Oct;26:e3061. DOI: http://dx.doi.org/10.1590/ 1518-8345.2631-3061

9. World Health Organization. Global strategy on human resources for health: workforce 2030. [Internet] Geneva; 2016 [cited 2020 Jan 29]. Available from: http://who.int/hrh/resources/pub_ globstrathrh-2030/en/

10. Neves T, Rodrigues V, Graveto J, Parreira P. Scale of adverse events associated to nursing practices: a psychometric study in Portuguese hospital context. Rev Latino-Am Enfermagem. 2018 Nov;26:e3093. DOI: http:dx.doi.org/10.1590/ $1518-8345.2595 .3093$

11. Almeida DB, Silva GTR, Freitas GF, Almeida IFB, Cunha ICKO, Amestoy SC. Freedom practices of nursing activists for the construction of other nursing objectification modalities. Rev baiana enferm. 2018;32:e25099. DOI: http:// dx.doi:10.18471/rbe.v32.25099

12. Downie R, Tannahill C, Tannahill A. Health promotion. Models and values. 2nd ed. Oxford: University Press; 2000.

13. Whithead D. Exploring health promotion and health education in nursing. Nurs Stand. 2018 Oct;33(8):e11220. DOI: http://dx.doi:10.7748/ ns.2018.e11220

14. Dempsey C, Barry M, Battel-Kirk B, CompHP Project Partners. Report Developing a European Consensus on Core Competencies for Health Promotion [Internet]. Paris: IUHPE; 2011 [cited 2020 Jan 29]. Available from: https://www. iuhpe.org/images/PROJECTS/ACCREDITATION/
European_Consensus_on_Core_Competencies_ for_HP.pdf

15. Jesus MC, Silva VA, Mota RS, Costa JCB, Mendes AS, Oliveira MJ. Repercussions of permanent education in the assistance practices of nursing professionals. Rev baiana enferm. 2019;33:e27555. DOI: http://dx.doi:10.18471/rbe. v33.27555

16. Xavier SPL, Pereira AP, Moreira MRC, Martins AKL, Ferreira HS, Machado MFAS. Competências em Promoção a Saúde à luz do Projeto Competencies Health Promotion (CompHP): uma revisão integrativa. Ciênc cuid saúde. 2019 Jan-Mar;18(1):e43421. DOI: http// dx.doi.org/10.4025/cienccuidsaude.v18i1.43421

17. Carvalho A, Carvalho G. Educação para a Saúde: conceitos, práticas e necessidades de formação. Lisboa: Lusociência; 2006.

18. Viana IS, Silva LF, Cursino EG, Conceição DS, Goes FGB, Moraes JMM. Education encounter of nursing and the relatives of children with special health care needs. Texto Contexto-Enferm. 2018;27(3):e5720016. DOI: http://dx.doi. org/10.1590/0104-070720180005720016

19. Ramos CF, Araruna RC, Lima CM, Santana CL, Tanaka LH. Education practices: research-action with nurses of Family Health Strategy. Rev Bras Enferm. 2018;71(3):1144-51. DOI: http://dx.doi: 10.1590/0034-7167-2017-0284

20. Santos JLG, Copelli FHS, Balsanelli AP, Sarat CNF, Menegaz JC, Trotte LAC, et al. Interpersonal communication competence among nursing students. Rev Latino-Am Enfermagem. 2019;27:e3207. DOI: http://dx.doi.org/10.1590/ $1518-8345.3226 .3207$

21. Ferreira FM, Brito IS, Santos MR. Health promotion programs in higher education: integrative review of the literature. Rev Bras Enferm. 2018;71(4):1814-23. DOI: http://dx.doi:10.1590/0034-7167-2016-0693

22. Freire RM, Landeiro MJ, Martins MM, Martins T, Peres HH. Taking a look to promoting health and complications' prevention: differences by context. Rev Latino-Am Enfermagem. 2016 Aug;24:e2749. DOI: http://dx.doi:10.1590/1518-8345.0860.2749

23. Nunes JM, Martins AKL, Nóbrega MFB, Souza AMS, Fernandes AFC, Vieira NFC. Promoting health in the hospital from the viewpoint of the nurse: descriptive-exploratory study. Online Brazilian J Nurs. 2019;8(3). DOI: https://doi. org/10.5935/1676-4285.20092568 
24. Netto L, Silva KL. Reflective practice and the development of competencies for health promotion in nurses' training. Rev esc enferm USP [online]. 2018;52:e03383. DOI: http://dx.doi. org/10.1590/S1980-220X2017034303383

25. Cassiani SHDB, Wilson LL, Mikael SSE, Morán-Peña L, Zarate-Grajales R, McCreary LL, et al.
The situation of nursing education in Latin America and the Caribbean towards universal health. Rev Latino-Am Enfermagem. 2017;25:e2913. DOI: http://dx.doi.org/10.1590/1518-8345.2232.2913

Recebido: 29 de janeiro de 2020 Aprovado: 22 de maio de 2020

Publicado: 9 de julho de 2020

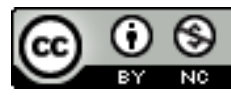

A Revista Baiana de Enfermagem utiliza a Licença Creative Commons - Atribuição-NãoComercial 4.0 Internacional.

https://creativecommons.org/licenses/by-nc/4.0/

Este artigo é de acesso aberto distribuído sob os termos da Licença Creative Commons (CC BY-NC).

Esta licença permite que outros remixem, adaptem e criem a partir do seu trabalho para fins não comerciais. Embora os novos trabalhos tenham de lhe atribuir o devido crédito e não possam ser usados para fins comerciais, os usuários não têm de licenciar esses trabalhos derivados sob os mesmos termos. 